\title{
A Characterization of Actuation Techniques for Generating Movement in Shape-Changing Interfaces
}

\section{Faisal Taher, John Vidler \& Jason Alexander}

To cite this article: Faisal Taher, John Vidler \& Jason Alexander (2016): A Characterization of Actuation Techniques for Generating Movement in Shape-Changing Interfaces, International Journal of Human-Computer Interaction, DOI: 10.1080/10447318.2016.1250372

To link to this article: http://dx.doi.org/10.1080/10447318.2016.1250372

Accepted author version posted online: 21

Oct 2016.

Submit your article to this journal $[\pi$

Џll Article views: 17

Q View related articles $₫$

View Crossmark data \lceil 


\title{
A Characterization of Actuation Techniques for Generating Movement in Shape-Changing Interfaces
}

\author{
Faisal Taher, John Vidler, and Jason Alexander. \\ School of Computing and Communications \\ Lancaster University
}

Lancaster, United Kingdom

f.taher@lancaster.ac.uk,j.vidler@lancster.ac.uk, j.alexander@lancaster.ac.uk

\section{A Characterization of Actuation Techniques for Generating Movement in Shape-Changing Displays}

\begin{abstract}
This article characterizes actuation techniques for generating movement in shape-changing displays with physically reconfigurable geometry. To date, few works in Human Computer Interaction literature provide detailed and reflective descriptions of the implementation techniques used in shape-changing displays. This hinders the rapid development of novel interactions as researchers must initially spend time understanding technologies before prototyping new interactions and applications. To bridge this knowledge gap, we propose a taxonomy that classifies actuator characteristics and simplifies the process for designers to select appropriate technologies that match their requirements for developing shapedisplays. We scope our investigation to linear actuators that are used in grid configurations. The taxonomy is validated by (a) examining current implementation techniques of motorized, pneumatic, hydraulic, magnetic, and shape-memory actuators in the literature, (b) constructing prototypes to address limited technical details and explore actuator capabilities in depth, (c) describing a use-case scenario through a case study that details the construction of a $10 \times 10$ actuator shape-display, and (d) a set of guidelines to aid researchers in selecting actuation techniques for shape-changing applications. The
\end{abstract}


significance of our taxonomy is twofold. First, we provide an original contribution that enables HCI researchers to appropriately select actuation techniques and build shapechanging applications. This is situated amongst other past works that have investigated broader application scenarios such as a shape-changing vocabulary, a framework for shape transformations, material properties, and technical characteristics of various actuators. Second, we carry out in-depth investigations to validate our taxonomy and expand the knowledge of vertical actuation in shape-changing applications to enable rapid development.

Keywords: Shape-changing displays, shape-changing interfaces, actuation techniques, taxonomy, case study, electromechanical, electromagnetic, shape-memory alloy, piezoelectric, pneumatic, hydraulic.

\section{Introduction}

Shape-changing interfaces are receiving increased attention within the Human-Computer Interaction (HCI) community due to their ability to augment user interaction through physicallydynamic surfaces (e.g. by transforming a flat display into physically protruding buttons). The HCI community has deployed a range of prototypical systems to examine user behaviour, interactions, and applications. At the heart of these systems are actuators that create physical motion. Several research prototypes have explored actuation using electro-mechanical motors (Leithinger and Ishii, 2010; Alexander, Lucero, and Subramanian, 2012; Follmer, Leithinger, Olwal, Hogge, and Ishii, 2013), pneumatic and hydraulic cylinders (Goulthorpe, Burry, and Dunlop, 2001; Wagner, Lederman, and Howe, 2002), Shape-Memory Alloy (SMA) wires (Nakatani et. al., 2005), electromagnets (Niiyama and Kawaguchi, 2008) and piezoelectric crystals (Hernandez, Preza, and Velazquez, 2009; Kyung et. al., 2011).

Despite the volume of shape-display research, there is limited reflective discussion regarding the specific actuator hardware configuration and characteristics. This makes it more 
difficult for new researchers to reason about the technologies that would enable them to make informed actuation decisions. There is, for instance, limited discussion about the relationships between the actuation force, speed, size footprint, granularity, and control system architecture specific to shape-changing applications. Previous characterization research has focused on establishing a shape-changing vocabulary (Rasmussen et. al., 2012), developing a framework for shape transformations (Roudaut et. al., 2013), exploring material properties (Coelho and Zigelbaum, 2011), and the technical characteristics of the various actuation techniques (Hollerbach, Hunter, and Ballantyne, 1992) in broader application scenarios. This article builds on this work and provides original contributions by focusing on actuation techniques used for vertical motion (i.e. linear actuators that are typically used in grid configurations) and their capabilities to inform researchers in HCI to appropriately select actuators for their applications.

This article therefore makes the following contributions: (1) A taxonomy that categorizes the key characteristics of linear actuators to aid shape-changing application development; (2) A literature review of shape-changing applications, the actuation techniques that were used, and how they were configured, (3) The construction of our own actuators based on these techniques to address the limited technical details provided by past shape-change literature, and to form a deeper understanding of their capabilities; (4) A case study that reflects on the development of a $10 \times 10$ grid of motorized actuators for a shape-changing bar char; (5) Guidelines to aid researchers to select actuators for shape-changing applications. We focus on vertical actuation due to their prevalence in shape-display literature and to scope our investigations.

The next section presents the Taxonomy of actuation techniques and is presented early to provide a reference point for the characteristics that we focus on during the literature review of 
vertical actuation in shape-changing displays, the construction of our own actuators, and the case study.

\section{Taxonomy for Vertical Actuation}

The characteristics of actuators can be described by a number of attributes; speed of actuation, granularity, holding torque or power, footprint or actuator size, complexity of control, and finally feedback method. Speed, power, and footprint attributes are informed by examining the range of values in past shape-changing applications and our lab prototypes (see Table 1). Granularity, control complexity and feedback method consist of typically known values. The attributes are broken down into categories, including a key within parentheses that are used in Table 1.

\subsection{Speed of Actuation}

The maximum speed of an actuator is often defined not by the actual drive type itself, but by the mechanical linkages packaged with the drive. This is especially evident in the case of stepper and DC motor drives where, with a lead screw, the speed of actuation can range from very slow to extremely fast, depending on the thread type used. Other linkages such as belt drives offer increased maximum actuation speed at the expense of force. In contrast, piezoelectric actuators have very slow moyement speeds, whereas pneumatics tends to have very high speeds. Based on these features, we characterize actuator speed as: (S) Slow - Less than $10 \mathrm{~mm} / \mathrm{second}$, (M) Medium - Between 10 and 50mm/second, and (F) Fast - Greater than 50mm/second.

\subsection{Granularity}

Granularity refers to the number of stable states (or vertical positions) that an actuator is capable 
of attaining. We use granularity, rather than precision, for actuation as it can be expressed as a number of possible states. Precision on the other hand must be described in terms of distance or angle, which is thus a complex compound characteristic involving the feedback method, linkage and the actuator type itself. Some actuators have mechanical linkages that inhibit high granularity positioning through losses in the linkage, or by the inability of the output to reach certain states. A belt-output stepper motor, for example, has predefined minimum stepping distances mechanically defined by the specification of the stepper motor and the radius of the driven wheel. Control electronics also further refine the ability of a stepper motor to accurately position itself. Micro-stepping the motor can allow higher resolution steps than the motor is able to achieve under normal circumstances. Other forms of actuator have much lower granularity; solenoids generally only have 2 states: fully extended and fully retracted. These states are almost always exactly at the minimum and maximum of the stroke length of the solenoid, barring more exotic control electronics. Thus we categorize the granularity of an actuator as: (L) Low Minimal granularity with 2 stable states, (M) Moderate - 3 to 1000 stable states along the length the actuator can travel, and (H) High - Many thousands of stable states along the actuator range of travel.

\subsection{Actuation Force}

Depending upon the specific application the actuator is intended for, the output force requirements may change to a great degree. Unfortunately, it is usually this same attribute that suffers as the size of an actuator decreases. Compounding this, the drive system beyond the actuator itself plays a large part in the final force at the display 'surface'. The range of strengths available to designers is vast, but to simplify the categorization of the ones we review, we divide the possibilities in to the following groups, designed to characterize the approximate typical 
strength of these technologies: (L) Low - less than 100g at the actuator output, (M) Moderate approximately $100 \mathrm{~g}$ to $1 \mathrm{~kg}$ at the actuator output, and $\mathbf{( H )}$ High - more than $1 \mathrm{~kg}$.

\subsection{Actuator Size (or Footprint)}

We classify actuator size by the area it occupies when looking along the axis of movement, with only the control equipment required to be co-located at the actuator itself. Piezoelectric actuators, for example, can be made in very small sizes, but the electronics to drive them can be many times larger than the actuator itself. With this in mind, we divide the actuators in to the following groups: (T) Miniature - less than $10 \mathrm{~mm}^{2}$, (S) Small - between 10 and $100 \mathrm{~mm}^{2}$, (M) Medium between 100 and $1000 \mathrm{~mm}^{2}$, and $(\mathbf{L})$ Large - more than $1000 \mathrm{~mm}^{2}$.

\subsection{Complexity of Control}

Shape-changing displays must consider the complexity of driving an actuator; some require complex systems of pipework to function properly (hydraulic or pneumatic), whereas others can be directly controlled by a microcontroller (DC motors or steppers). Notably, the size of the controlling equipment does not always scale proportionately with the size of the actuator; many small stepper motor driver chips are able to operate many different sizes of stepper motor. Equally, small electromagnets and SMA wires require large external power supplies. The required equipment to control a pneumatic system is significant for a low number of actuators, but becomes less significant as the number increases, due to component reuse (reservoirs, regulators, etc.). We classify the various scales of control equipment as: (S) Simple - Those systems which can be directly driven from the controlling logic, (M) Moderate - Actuators requiring intermediate controlling equipment, but typically with a direct relationship with the 
controller, and (C) Complex - Requiring large numbers of additional control and supply parts, and safety equipment.

\subsection{Feedback Method}

Some types of actuator lend themselves to particular styles of control - linear screw drives can be easily mated with linear potentiometers for PID control, for example - whereas others do not gain significant benefits to using certain approaches - solenoids, for example, can simply be limit switched, or just assumed to have worked as expected (open loop control). These forms of control break down as: (D) Direct- the position is guaranteed for a given signal without feedback, (CL) Closed Loop - drives which need to be placed in a closed-loop feedback system to maintain accurate positioning, e.g. using PID/PD/PI control ${ }^{1}$, and (OL) Open Loop - drives which are predictable enough to move to known states at every control impulse.

\subsection{Taxonomy Summary}

This taxonomy categorizes the key properties of actuators suitable for inducing vertical movement. The next section uses these properties to examine actuator use in shape-change in both previous literature (summarized in Table 1) and in our own exploration of prototypes. We then present a case study that examines challenges beyond the taxonomy, and discuss the relationships between the various categories.

\footnotetext{
${ }^{1}$ A PID controller computationally allows the moving part of an actuator to move to a desired position by
} taking into account its current position (e.g. by potentiometer feedback) and the difference between the desired and current positions. 


\section{Exploration of Actuation Techniques}

To provide an overview of vertical actuation techniques, we explore the actuator details provided by the literature in shape-changing applications along with our own prototypes, and define their characteristics as described in the taxonomy above (summarized in Table 1). We include our own prototypes to explore gaps in the literature and discuss the implications of using these actuators in shape-changing applications. Each prototype is a small-scale deployment (in terms of footprint) using each actuation technique to form a deeper understanding of their capabilities and characteristics. We provide representative examples of the actuator configurations to illustrate how to power an actuator and control its vertical position - there are other alternate control system designs, but we attempt to use the simplest possible design that allows finegrained control.

\subsection{Electro-mechanical Actuation}

\subsubsection{Background}

Electro-mechanical actuators (e.g. DC, Stepper, and Servo motors), generate motion through an interaction between magnets and coils of wire. Several shape-display prototypes utilize this approach. An early example is Surface Display (Hirota and Hirose, 1995). The system consists of $1600 \mathrm{~mm}^{2}$ actuators in a $4 \times 4$ grid capable of $50 \mathrm{~mm}$ stroke, $166.6 \mathrm{~mm} / \mathrm{sec}$ speed, and designed to explore interaction with virtual objects through a physically actuated medium. The actuators were controlled using a timer interface, microcontroller unit (MCU), and used closed loop control through a potentiometer. The FEELEX (Iwata, Yano, Nakaizumi, and Kawamura, 2001) shape-display consists of a grid of 23 servo-motors with projection based visual output on a flexible layer. The actuators can achieve a stroke of $80 \mathrm{~mm}$ at a speed of $100 \mathrm{~mm} / \mathrm{sec}$, apply 
110gf, and controlled by a parallel I/O port coupled with an optical encoder for position feedback. Wagner et. al. (2002) constructed a 6×6 PWM-controlled servomotor-based haptic display with actuating pins capable of $2 \mathrm{~mm}$ stroke, a speed of $41 \mathrm{~ms}$, and control circuitry that included a parallel I/O port. Tilt Displays (Alexander et. al., 2012) also uses servo motors to control a $3 \times 3$ grid of OLED displays with $9.1 \mathrm{~mm}$ stroke at $20 \mathrm{~mm} / \mathrm{sec}$, which are designed to physically augment $3 \mathrm{D}$ scenes. The servos have a $121 \mathrm{~mm}^{2}$ footprint and are controlled through a MCU. Microsoft's Physical Charts ${ }^{2}$ prototype is a $6 \times 2$ grid of stepper motors that control measuring tapes using custom built gears, to show bar chart data.

Relief and Sublimate (Leithinger, Lakatos, Devincenzi, Blackshaw, and Ishii, 2011; Leithinger et. al., 2013) contain 120 motorized potentiometers that can achieve a stroke length of $127 \mathrm{~mm}$ and driven by a MCU and motor driver. The user interface for Relief consists of projection on top of a flexible layer, whereas Sublimate explores an augmented reality based interface. The inFORM system (Follmer et. al., 2013) is a large-scale display that consists of 900 actuated polystyrene push-pull rods actuated by motorized potentiometers and uses projected visual output. The system consumes up to $2700 \mathrm{~W}$. Each actuator can exert $110 \mathrm{gf}$ and is controlled by a MCU. EMERGE (Taher et. al., 2015) consists of 100 motorized potentiometers capable of $100 \mathrm{~mm}$ stroke, $130 \mathrm{gf}$, actuate at $\sim 200 \mathrm{~mm} / \mathrm{sec}$, and controlled using Master/Slave MCUs and motor drivers. Maximum power consumption for each actuator is $8 \mathrm{~W}$. Each push rod connected to the motors contain an RGB LED.

2 http://research.microsoft.com/en-us/um/cambridge/projects/physicalcharts/ (29/06/2016). 
The BMW kinetic sculpture ${ }^{3}$ is designed as an art-piece, consisting of a matrix of 714 metal spheres over $6 \mathrm{~m}^{2}$ controlled by individual stepper motors. These spheres can rise and fall to display car models. ShapeClip (Hardy, Weichel, Taher, Vidler, and Alexander, 2015) uses a stepper motor and lead-screw configuration to vertically drive attachments. Each actuator has a $400 \mathrm{~mm}^{2}$ footprint capable of $60 \mathrm{~mm}$ stroke at $80 \mathrm{~mm} / \mathrm{sec}$. Each unit is powered with $2.4 \mathrm{~W}$ (including a portable battery-based alternative), able to produce $\sim 250$ grams of force, and controlled using a MCU and motor driver. It uses light to control height (e.g. from an LCD display) and is capable of 256 states (256 shades of RGB grayscale).

\subsubsection{Lab Prototypes}

We explored two types of motorized actuation: a DC motor (continuous revolution) and a stepper motor (known discrete revolutions). Although both motors operate in a similar manner (i.e. the Oersted effect causes the coils to turn the motor shaft - Oersted, 1820), they differ in that stepper motors do not require closed-loop control (where the position is continually read, e.g. via an encoder).

DC Actuator Configuration: We utilized a Bourns motorized linear potentiometer ${ }^{4}$ (referred to as a slider) for our setup (Figure 1) similar to the sliders used by inFORM (Follmer et. al., 2013), EMERGE (Taher et. al., 2015), and Relief (Leithinger et. al., 2011). The T-bar moves vertically through a mini gear and pulley, which are controlled by the motor shaft. As servomotors use this same configuration, but measures angular position rather than linear position, and uses gearing rather than a belt drive, this section is applicable to them as well. The slider is connected to an $\mathrm{H}$ -

$3 \mathrm{http} / /$ artcom.de/en/project/kinetic-sculpture/ (29/06/2016).

4 http://www.mouser.co.uk/ProductDetail/Bourns/PSM01-082A-103B2/ (29/06/2016). 
bridge motor driver (see Figure 1) and an ATTiny84 MCU which can accurately control its position (feedback provided by built-in potentiometer) at $\sim 8 \mathrm{MHz}$ using a proportional-integralderivative (PID) controller.

DC Actuator Characteristics: This particular slider is capable of $100 \mathrm{~mm}$ stroke. Larger and smaller strokes depend on the manufacturer and availability. The sliders are able to support $\sim 130 \mathrm{~g}$ of force and actuate at $\sim 200 \mathrm{~mm} / \mathrm{s}$. The size of each slider is $150 \mathrm{~mm}$ (length) $\times 50 \mathrm{~mm}$ (width) $\times 13 \mathrm{~mm}($ depth) and weighs $75 \mathrm{~g}$. Each slider is powered using a $10 \mathrm{~V}$ supply and can require up to $\sim 0.8 \mathrm{~A}$ (i.e. $8 \mathrm{~W})$.

Stepper Actuator Configuration: The stepper motor used was similar to those used in DVD drives. Attached to the motor is a lead screw that vertically moves an attachment (Figure 2). The stepper motor is controlled by a custom-built PCB that contains an ATmega328p MCU. Vertical position is controlled using the known number of steps.

Stepper Actuator Characteristics: Each stepper motor is designed to produce a $60 \mathrm{~mm}$ stroke using a lead screw. A longer lead screw can be attached to increase stroke length. This would consume additional power due to longer on-time. The lead screw pitch allows the motor to support $\sim 250 \mathrm{gf}$ and actuate at $80 \mathrm{~mm} / \mathrm{sec}$. The force and speed is dependent on the density and pitch of the lead screw, i.e. lower density enables faster actuation, and a lower pitch allows higher actuation force. Each motor is approximately $20 \mathrm{~mm}$ (width) $\times 20 \mathrm{~mm}($ depth $) \times 80 \mathrm{~mm}$ (height) whilst closed and weighs $30 \mathrm{~g}$. Each stepper motor unit requires up to $\sim 0.54 \mathrm{~A}$ at $5 \mathrm{~V}$ (i.e. $2.5 \mathrm{~W})$. 


\subsubsection{Summary and Implications for Shape-Changing Applications}

Both DC and stepper motor prototypes, with similar DC motors used in inFORM (Follmer et. al., 2013) and EMERGE (Taher et. al., 2015), can be stacked closely together as their physical configuration is relatively minimal. The built-in potentiometer of the DC motor prototype provides accurate closed-loop position control, thus enabling shape-changing applications to exploit height resolution (e.g. in data visualization applications where height corresponds to specific values). In contrast, an open loop method as used in the stepper motor approach allows for less accurate positioning; however it is possible to use a closed loop approach (e.g. through a potentiometer). The actuation speed is significantly higher with the DC motor prototype, and can therefore support higher refresh rates at the user interface level and transition between shapes.

In cases where higher vertical pixel resolution (i.e. bars stacked close together) is desired the size footprint is still too large at the actuator scale for both approaches and therefore requires mechanical linkages to reduce pixel spacing on the interface level. In such cases the lead screw of the stepper prototype is more beneficial as it is able to exert nearly twice as much force to support the weight and friction of linkages in comparison to this particular DC slider.

A key advantage for belt drives is that the gear/pulley system allows the slider to be backdriven (e.g. if users want to press an actuating pin/pixel), and allows smoother touch interactions. A lead-screw can be back-driven, but this depends on the thread pitch (a coarser thread pitch would be easier to press down) and back-drive is also less smooth. On the other hand, a gear/pulley belt drive is more susceptible to deterioration from long-term usage as there are more small moving parts involved. 


\subsection{Pneumatic Actuation}

\subsubsection{Background}

Pneumatic actuation converts energy using compressed air into mechanical motion. The Aegis Hyposurface (Goulthorpe et. al., 2001) is a large scale dynamically reconfigurable pneumatic surface designed as a reactive display and consists of 576 pistons capable of $500 \mathrm{~mm}$ stroke. The system uses metallic plates attached to the pistons with projected output. Gemotion (Niiyama and Kawaguchi, 2008) is a smaller scale pneumatic cylinder/piston based display designed to show 3D shapes using a flexible screen and projection. The system consists of a $7 \times 15$ grid of actuators capable of $150 \mathrm{~mm}$ stroke at $450 \mathrm{~mm} / \mathrm{sec}$. Harrison and Hudson (2009) experiment with dynamic physical buttons using pneumatics; changing between concave, convex, and flat states using latex layers and visual output from rear projection.

\subsubsection{Pneumatic Lab Prototype}

Actuator Configuration: We constructed a pneumatic actuator using a double acting cylinder, a linear slide potentiometer attached to the cylinders, a solenoid, and an electro-pneumatic pressure regulator (Figure 3). The components were purchased from VEX Robotics ${ }^{5}$ (pneumatics kit 2). We used an Arduino Mega2560 as a controller. The regulator controlled the airflow to the cylinder through a solenoid by mapping the voltage rating to the flow rate and directing air to the appropriate valve. These allowed a PWM signal to control the air flow rate. Transistors were used to drive the $10 \mathrm{~V}$ pneumatic switch and regulator, requiring an additional power source. We created a closed loop control system by attaching a linear potentiometer to the cylinder, which

5 http://www.vexrobotics.com/pneumatics.html/ (29/06/2016) 
enabled position feedback and PID control. To scale this configuration to multiple cylinders, we would require careful selection of tube diameter and regulator positioning to ensure sufficient pressure would be available.

Actuator Characteristics: The cylinder is capable of $50 \mathrm{~mm}$ stroke. Larger and smaller strokes depend on the manufacturer and availability. Each cylinder holds a maximum pressure of $100 \mathrm{psi}$ and produces up to $5.4 \mathrm{~kg}$ of force at a speed of $\sim 254 \mathrm{~mm} / \mathrm{sec}$. The reservoir supports $150 \mathrm{ml}$ of compressed air. With the attached components (i.e. potentiometer and linkages) each pneumatic actuation is $150 \mathrm{~mm}$ (closed height) $\times 40 \mathrm{~mm}($ width $) \times 25 \mathrm{~mm}($ depth $)$ and weighs $20 \mathrm{~g}$. The regulator is powered by $\sim 26 \mathrm{~V}$ and $\sim 0.05 \mathrm{~A}$ (i.e. $1.04 \mathrm{~W}$ ) and requires up to $10 \mathrm{~V}$ for an input signal (i.e. the pressure valve opening is proportional to the input signal). The solenoid requires $5 \mathrm{~V}$, 0.05A (i.e. 0.4 Watts). In total, a single cylinder requires $\sim 1.5 \mathrm{~W}$. An air compressor would also be required for continuous air supply, which can consume a high amount of power (up to 100W).

\subsubsection{Summary and Implications for Shape-Changing Applications}

The pneumatic actuator can exert high force, which can be useful for supporting mechanical linkages to increase pixel resolution at the interface level, given the actuator size footprint is relatively large. The actuation speed is high and can therefore support high interface refresh rates (transitioning between shapes). It supports smooth back-drive, which allows users to smoothly press and pull the attached pin. However, the physical configuration requires several components (i.e. solenoids, regulators, switches), which increases the overall size footprint and complexity of the setup. In addition, the air tubes can suffer from leaks (e.g. if valves are not air-tight).

Whilst the closed loop control is similar to the motorized slider, the PID controller needs to be adjusted to cater for air pressure losses each time the length or size of the tubing is altered 
(e.g. if longer tubing is need to reach actuators). This increases the complexity of application development. Furthermore, position control requires careful consideration as issues such as static friction and compliance can cause inaccuracies. Gemotion (Niiyama and Kawaguchi, 2008), for instance, used a friction damper to improve the accuracy of their pneumatic piston.

\subsection{Hydraulic Actuation}

\subsubsection{Background}

Hydraulic actuation is built on the principle of manipulating fluid to create mechanical motion. Tactus is a commercially available technology that can show raised buttons on a touch-screen display through hydraulic actuation ${ }^{6}$. The tactile layer is a flat overlay that sits on top of a touch sensor and deforms into buttons or shapes of specific height, size, and firmness which users can feel and press down. The tactile layer replaces the passive glass layer of a touchscreen and is made of thin multi-layer stacks of polymers. The topmost layer is an optically clear elastomer, beneath which are several micro channels where fluid can flow.

\subsubsection{Hydraulic Lab Prototype}

Actuator Configuration: Our hydraulic setup (purchased from $\mathrm{RC}_{4} \mathrm{WD}^{7}$ starter kit system 1) consisted of a hydraulic cylinder (Figure 4), a motor and pump, a fluid reservoir (with ISO32 viscosity rating), and an Electronic Speed Controller (ESC). An Arduino UNO was connected to the pump ESC. To receive position feedback, we attached a linear potentiometer to the cylinder. Similar to our pneumatic setup described above, this allowed us to use PID control to drive the

$6 \mathrm{http} / /$ tactustechnology.com/ (29/06/2016).

7 http://store.rc4wd.com (29/06/2016). 
cylinder. Unfortunately, our setup was limited to a unidirectional stroke due to part availability; however bidirectional actuation can be achieved with a double acting pump or through additional valves.

Actuator Characteristics: The cylinder is capable of a $75 \mathrm{~mm}$ stroke, which is dependent on the manufactured unit. The hydraulic cylinder is able to support $80 \mathrm{~kg}$ (significantly more than other actuation techniques given its size footprint) and moves at a rate of $\sim 18 \mathrm{~mm} / \mathrm{sec}$. The cylinder is $20 \mathrm{~mm}$ in diameter with the potentiometer fitting and $135 \mathrm{~mm}$ in height (whilst closed). This configuration requires a $12 \mathrm{~V}, 22 \mathrm{~A}$ power supply, or $264 \mathrm{~W}$ for each actuator.

\subsubsection{Summary and Implications for Shape-Changing Applications}

Using hydraulics for vertical actuation in shape-display applications is relatively uncommon; Tactus being an example of vertical actuation through a small "bump". The construction of hydraulic components can be "messy" due to working with oil-based fluid. Similar to pneumatic systems, hydraulics can also suffer from leakages, thus the physical setup needs to be robust to support the high force generated from the piston and pump (the support mechanisms subsequently increase the footprint).

The actuator can support higher loads (e.g. heavy linkages), however the actuation speed is very low, which would make transitioning between shapes on the user interface non userfriendly. This actuator has no option to back-drive, unless specially designed equipment is used. Therefore, touch interaction is limited. Input power is also exceptionally high compared to other approaches. Miniaturized actuators that can be stacked in a grid matrix is proposed and discussed by the Digital Clay concept (Zhu and Book, 2004), including actuator design, feedback methods (based on non-contact resistance using a conductive ring and metal film on the outer surface of 
the cylinder), and approaches for control architecture (e.g. similar to driving an LED array).

\subsection{Smart Materials}

\subsubsection{Background}

Smart materials are capable of altering their properties via external stimuli, such as an electric current. Shape-Memory Alloys (SMAs) are common materials used in shape-display applications. Lumen (Poupyrev, Nashida, Maruyama, Rekimoto, and Yamaji, 2004) consists of a $13 \times 13$ pixel grid composed of plastic tubes lit with LEDs. Surflex (Coelho, Ishii, and Maes, 2008) uses flexible foam with embedded circuits and SMAs as soft pixels (1076 $\mathrm{mm}^{2}$ footprint) that can deform into different shapes. Nakatani et. al. $(2003,2005)$ investigated a SMA-based set-up using a $4 \times 4$ pin-rod matrix, which achieves $120 \mathrm{~mm}$ stroke. The actuators are capable of exerting 30gf, actuate at $1.5 \mathrm{~mm} / \mathrm{sec}$ and are controlled by an FET switch and photo reflectors for position feedback. A second prototype was also built as a $16 \times 16$ pin matrix using $4 \mathrm{~mm}$ $($ diameter $) \times 30 \mathrm{~mm}$ (height) cylindrical rods at $5 \mathrm{~mm}$ spacing and able to actuate at $1 \mathrm{~Hz}$. This approach used an oil-based cooling mechanism and a camera/marker-recognition method to provide closed-loop position feedback.

\subsubsection{Shape-Memory Alloy Lab Prototype}

Actuator Configuration: An SMA based actuator was constructed using a Nickel-Titanium spring ( 0.75 wire diameter) and a metal extension spring (Figure 5). These were attached on both ends of a $60 \mathrm{~mm}$ height linear potentiometer. The compression of the SMA by applying current and the competitive force from the metal springs enabled vertical movement. The SMA actuator position is controlled by switching a power supply to the SMA through a MOSFET transistor. 
The slow cooling process of the SMA at room temperature means that the actuator will move faster in one direction, therefore an active cooling system (e.g. a cooling fan) must be directed towards the SMA to enable equal bidirectional movement speed. As the position is a function of the SMA temperature, maintaining a specific level of heat requires careful interplay between cooling and heating.

Actuator Characteristics: The actuator is only capable of a $6 \mathrm{~mm}$ stroke, but this is highly dependent on the properties of the SMA. A thinner coil would allow faster actuation at the expense of strength, and vice versa. Several variants of metal springs were tried (i.e. different diameters and lengths). The SMA spring is capable of $\sim 400 \mathrm{gf}$, and can actuate at a rate of $\sim 1.03 \mathrm{~mm} / \mathrm{sec}$. The dimensions of the actuator are $20 \mathrm{~mm}$ (width) $\times 20 \mathrm{~mm}($ depth $) \times 80 \mathrm{~mm}$ (height) and weighs $18 \mathrm{~g}$. Once the SMA spring is extended using the full force of the metal extension spring, a total current of $3 \mathrm{~A}$ is required with $3.3 \mathrm{~V}$ (i.e. $9.9 \mathrm{~W}$ ), in order to heat up the SMA through resistive heating such that it returns to its original compressed shape.

\subsubsection{Summary and Implications for Shape-Changing Applications}

The SMA prototype has low actuation speed, therefore creating a slow refresh rate (e.g. for transitioning between shapes on the interface level). It also requires additional equipment to carefully control heating and cooling. For example, Nakatani et. al. $(2003,2005)$ experimented with a cooling fan, oil (combined with Peltier effect element), and ethylene glycol. Extension springs used for applying competitive force are likely to suffer from 'learning' the extended position through multiple extensions by the SMA spring. One approach is to replace the extension spring with a second SMA spring; however this increases power requirements and 
requires additional cables. The heat required to actuate the SMA spring (up to $50^{\circ} \mathrm{C}$ ) risks damage to the potentiometer.

This particular SMA prototype has a medium size footprint and can therefore be stacked closely together. Mechanical linkages can be adequately supported as SMA can exert $\sim 400$ gf to increase resolution on the interface. Nakatani et. al's (2005) 3D form display already consists of a small footprint of $25 \mathrm{~mm}^{2}$ per actuator, therefore creating a high resolution interface. Beneath the interface level, external cooling and safety mechanisms increases the size and control complexity. The reliance on temperature also causes difficulties in achieving high accuracy in position control (Nakatani et. al., 2005), which can be problematic in applications such as data visualization where height corresponds to values. Power consumption is high for our prototype, i.e. nearly $10 \mathrm{~W}$ without cooling, whereas the $3 \mathrm{D}$ form displays consumes $3.9 \mathrm{~W}$ per actuator with oil-based cooling. In terms of back-drive, rods attached to the SMA prototype can be smoothly pressed down.

\subsection{Electro-magnetic Actuation}

\subsubsection{Background}

In addition to driving electric motors, prototypes have used magnetism to generate motion in different ways. Prototypes such as SnOil (Frey, 2004) use the magnetic field to manipulate fluid (i.e. magnetorheological ER fluid, Ferrofluid). Consisting of a grid of $12 \times 12$ electromagnets with a basin of Ferrofluid that rests on top, the Ferrofluid reacts to the fields to create 'bump'like structures. The electromagnets are powered via multi-layered printed circuit boards. FriskenGibson, Bach-y-Rita, Tompkins, and Webster (1987) constructed a haptic display aimed for blind users; an $8 \times 8$ array of mini solenoids capable of 4 linear positions controlled using an ADC 
converter and solenoid controller.

\subsubsection{Electro-magnetic Lab Prototype}

Actuator Configuration: We developed a magnetic actuator by using an electromagnetic coil, a cylindrical neodymium magnet, an H-bridge motor driver, and linkages that allow a push rod to move vertically by pseudo-levitation (Figure 6 ). The electromagnet was encased in a 3D-printed unit and the neodymium magnet housed in a Teflon tube, which allowed low friction movement of a plastic push rod. Positioning was controlled by PWM, which correlates to the strength of the magnetic field repulsion and thus proportional to the height of the repelled magnet. Unlike with a motor, on-time does not equate to the actuation pin moving up or down. This meant that maintaining a specific position required the electromagnet to constantly be switched on, consuming a large amount of power and heating the electromagnet. An H-bridge capable of handling the high voltage and current levels, polarity reversal, and efficient handling of PWM signals was used.

Actuator Characteristics: The stroke length is $17 \mathrm{~mm}$. The current setup uses a plastic push rod, $2 \mathrm{~g}$ in weight as in the pseudo-levitation approach, additional weight reduces the stroke capacity of the magnet. The speed of movement was $\sim 85 \mathrm{~mm} / \mathrm{sec}$. Each actuator was $\sim 30 \mathrm{~mm}$ in diameter and $\sim 150 \mathrm{~mm}$ in height, and weighed $70 \mathrm{~g}$. Each electromagnet consumes $\sim 30 \mathrm{~V}$ and $\sim 0.6 \mathrm{~A}$ (i.e. $18 \mathrm{~W}$ during peak).

\subsubsection{Summary and Implications for Shape-Changing Applications}

Heat generation was a prevalent issue with our prototype, which becomes hot after $\sim 30$ seconds of operation at $30 \mathrm{~V}$. A cooling system such as a fan and a heat-sink (e.g. a steel baseplate similar to ForceForm - Tsimeris, Dedman, Broughton, and Gedeon, 2013) is essential for continuous actuation. Thus, although the physical requirements are relatively minimal, safety measures such as cooling increases the footprint (similar to the SMA actuation approach described above). 
Power consumption is high, and the use of levitation means that the electromagnet must remain powered to maintain a specific position. Search Display (Frisken-Gibson et. al., 1987) uses solenoids as linear actuators with four heights; however it is unclear whether heat dissipation during continuous usage required cooling. Similarly, the electromagnets used in SnOIL (Frey, 2004) need enough charge to create a small "bump" in the Ferrofluid but the input power and cooling details are not provided.

Once the magnet is repelled in our prototype, the weight it can support decreases proportional to the square of the distance, thus creating low force output. Therefore, mechanical linkages in increase interface-level resolution would be impractical. Stacking the actuators also requires insulation to prevent magnetic interference, which also increases the size footprint. Back-drive is feasible and any mechanical attachment would also automatically return to its original position. Height resolution is adequate as the PWM signal is proportional to how far the mechanical attachment actuates, which also allows accurate control.

\subsection{Piezoelectric Actuation}

\subsubsection{Background}

Piezoelectric actuation is typically used for sub-millimeter, high-precision applications. Piezo expands when an electric current is applied, and typically, piezo actuators have been used for purposes such as vibration feedback on interactive displays (e.g. Poupyrev et. al., 2002; Rekimoto and Schwesig, 2006; Chauvelin et al., 2014) or embedded into input devices such as mice (Kyung, Kwon, and Yang, 2010). The TAXEL system (Kyung et. al., 2011) explores deformable touch screens with projected visuals by using a thin-film piezoelectric actuator, which is $36 \mathrm{~mm}^{2}$ in size, able to exert $51 \mathrm{gf}$ and controlled using a switch coupled with photo 
reflectors for position feedback. Hernandez et. al. (2009) explore a custom made miniature piezoelectric linear actuator for braille output $\left(9 \mathrm{~mm}^{2}\right.$ footprint), consisting of a piezo-ceramic disk attached to a shaft and slider, which achieves vertical movement through vibration and inertia, and controlled using a master and slave MCUs.

\subsection{Actuation Techniques Summary}

The descriptions and classification (with our taxonomy) of shape-changing displays in past literature has exposed gaps in actuator descriptions (particularly speed, force and size footprint) and reflective discussions of their shape-changing applications. The lab prototypes aimed to provide more detail in terms of actuators. However, shape-display applications are very specific and can require variable numbers of actuators in various arrangements. The build-process becomes difficult to predict and classify due to application-specific requirements. To better understand the challenges faced in specific shape-changing applications and to extend our characterization beyond the taxonomy, we describe a case study in the next section that details the implementation of a physically dynamic bar chart. We discuss the challenges faced and provide guidance for future implementations of similar systems.

\section{Case Study: Shape-changing Bar Chart}

Our goal was to develop a physically dynamic bar chart with self-actuating and back-drive capable bars; integrating a commonly used actuation style and providing a control system at scale. Users should also be able to physically interact with the bars to perform a range of data analysis tasks. The hardware requirements included (1) fast, smooth, and accurate actuation, (2) overall size that made it usable whilst standing, (3) appropriate bar spacing which allowed users 
to comfortably touch and interact with the bars, and (4) can be continuously used over medium periods of time. Below, we describe the actuation technique according to our taxonomy, the hardware assembly process, and the control architecture (a full system description can be found in: Taher et. al., 2015). We decided to use the motorized linear potentiometers as described above in our explorations of actuation techniques. The properties of the sliders can be classified using our taxonomy as: Fast actuation speed, Medium/High granularity, Moderate actuation force, Small size footprint, Moderate control complexity, and PID feedback method.

\subsection{Assembly}

A $10 \times 10$ configuration allowed a manageable size to construct as a first prototype, and also provided an adequate range for showing bar chart data. The actual bars of the bar chart consisted of $9.5 \mathrm{~mm}^{2}$ plastic push rods. An important challenge was to achieve adequate push rod spacing for users to interact with. If the push rods were placed directly above the sliders, they would be $>20 \mathrm{~mm}$ apart, which would hinder user experience. By introducing mechanical linkages (i.e. plastic tubing and rod guides) and by arranging the sliders in two levels (Figure 7), we reduced the spacing to $10 \mathrm{~mm}^{2}$. However, the additional weight and friction from the linkages slowed down the slider actuation speed (which is limited to supporting $130 \mathrm{~g}$ ).

To compensate for additional weight, we overdrove the motors using $12 \mathrm{~V}$ (exceeding the $10-11 \mathrm{~V}$ rating). This caused the setup to require up to $\sim 960 \mathrm{~W}$ when all the sliders are switched on, which created a large input power footprint. To allow low friction movement in the linkages, we utilized Teflon tubing (known for their lubricity) to guide flexible fiber-optic rods from the sliders to the plastic push rods. The mechanical linkages also increased the overall height and weight of the system, as we had to use 4 layers of $400 \mathrm{~mm}^{2} \times 5 \mathrm{~mm}$ (thickness) Perspex to hold 
the 100 Teflon tubes and fiber-optic rods in place. A structure of aluminum bars provided rigid support for the entire setup, which was necessary when all sliders are switched on and generating large amounts of movement. The vertical distance between the sliders and the push rods had to be carefully considered, as larger angles to bring the push rods closer together increased friction.

\subsection{Visual Output}

The visual output of each actuating bar allows users to differentiate between data points, rows, and columns. We chose to illuminate each bar with an RGB LED, which can be individually addressed by the control system. The placement of the LEDs required careful planning to avoid overlapping colours, adequately lighting of each bar, and avoiding additional weight from linkages. We decided to place 10 LED strips (one strip contains 10 LEDs) on 10 3D printed column strips that were placed directly underneath the top panel of the system (these also served as guides for the plastic bars). To enhance the light from the LEDs along the length of the plastic bars, we experimented with using optical fibre tubes inside the bars, however, this slowed down actuation from the extra weight. Therefore, we sanded the bars to create a frosted effect, which in turn adequately distributed the light.

\subsection{Control architecture}

To enable each slider to be individually addressable, we constructed 17 custom driver boards that consisted of 3 ATTiny 84 MCUs and 6 motor drivers that were able to drive 6 sliders using PID control. The driver boards were connected by an SPI bus and controlled by 2 Arduino Mega2650s. One issue that we faced was the electrical noise from closely stacked data cables that caused fluctuations in potentiometer position feedback readings. The synchronization of the 
various MCUs (i.e. 2 Arduinos and 52 ATTinys) also required careful consideration as it was important to achieve a high data-bandwidth, i.e. to display sample and refresh rate.

\subsection{Reflection}

The demands of a physically dynamic bar chart caused unexpected implementation challenges despite selecting the most suited actuation technique for our shape-changing application; in particular, applying mechanical linkages whilst maintaining fast actuation and stacking 100 actuators in close proximity. CAD software such as AutoDesk Inventor confirmed the feasibility of the configuration, but understanding the interaction between different materials (e.g. friction, robustness) required a trial-and-error approach. For instance, we experimented with various combinations of push rods and tubing before selecting fiber-optics and Teflon. In addition, we found that accurately controlling 100 closely stacked actuators requires shielding from electrical noise.

We chose motorized potentiometers as they included several built-in components, such as position feedback for accurate closed-loop control, a motor for driving the plastic bars of EMERGE, and the ability to develop push and pull interactions (enabled by the belt-drive approach) in our shape-changing application. A key challenge in controlling the sliders was developing a custom PID controller, which had to be adjusted due to the weight from mechanical linkages.

The actuators and materials used in constructing EMERGE were fairly robust, as only three sliders have required replacing (due to detached belts) over a two-year period with regular usage. The faults were caused by a combination of the strain on the gear and pulley components from the linkages, the fast actuation speed (i.e. $200 \mathrm{~mm} / \mathrm{sec}$ ) from the increased voltage input (to support linkages), and the way in which users interact with the bars. Whilst high speed actuation 
is useful (e.g. to transition between shapes faster), it is important to slow down actuation speed where appropriate (e.g. for certain interactions) to reduce the strain on the sliders. As a result, the maintenance of the various components need careful consideration, i.e. damaged sliders must be easily accessible. In such cases a more modular approach would be beneficial to minimize time on disassembling linkages. With regards to user interaction, we found that some users are gentle whilst pushing and pulling the bars, others can be forceful (which causes strain on the gear and pulley components).

\section{Discussion}

Selecting an actuation technology for developing shape-displays is a matter of balancing desired features against their side effects; ultimately these choices will depend on the applicationspecific requirements. Below we discuss these tradeoffs in the context of requirements surrounding actuator prototyping, resolution, density, interaction, visual output, and power. These are supported by guidelines to help researchers select actuation techniques for shapechanging applications.

\subsection{Prototyping Shape-Changing Displays}

Electromechanical actuation techniques (e.g. stepper motor, DC motor, servo motor) are better suited to developing low-fidelity prototypes, i.e. actuators that are functional with coarse grain control (e.g. the ability to move up and down), and are designed for experimentation. This is due to the simplicity in configuring a motor to vertically actuate, as well as their high commercial availability. For instance, a motorized potentiometer (e.g. as used in our prototype investigations in section 3.1.2) can be controlled with a motor driver and microcontroller. Actuators based on 
fluids, such as hydraulic and pneumatic, require a learning curve in terms of fluid dynamics and a more complex set up of reservoirs, tubing, solenoids, and pumps. Electromagnetic and SMA techniques are simpler than fluid-based techniques as they require a current to activate, however the vertically moving part needs to be custom built.

High-fidelity prototypes with accurate control that are designed for reliable and continuous usage can range from electromechanical, hydraulic to pneumatic techniques. In effect, using pneumatics can actually be more reliable than electromechanical techniques as there are less moving parts (i.e. using cylinders in fluid systems compared to gears, lead screws and belt-drives that are more susceptible to wear and tear). Electromagnetic and SMA approaches are less suited to high-fidelity systems as they are more likely to overheat rapidly and require sophisticated cooling mechanisms. For example, Nakatani et. al's SMA prototype $(2003,2005)$ included submerging the actuators in an oil based cooling reservoir.

Guideline 1: Electromechanical actuation techniques are best suited for constructing low-fidelity shape-display prototypes as they are easy to configure and re-configure. While electromechanical actuators are also suited for high-fidelity prototyping, pneumatic and hydraulic techniques can be more reliable as they contain less moving parts.

\subsection{Shape-Changing Display Resolution and Density}

Shape-display resolution (i.e. the number of actuating components that users can interact with) and density go hand-in-hand, as developing a high resolution display requires a high number of closely stacked actuators. Actuators that are far apart can hinder user experience, and show an inadequate level of detail. To achieve higher resolution at the interface level, actuators in shapechanging displays typically use mechanical linkages to condense the output area. However, these linkages create additional weight and friction which slows down actuation speed and reduces the 
final power output, especially for electro-mechanical actuators with limited torque. Pneumatic and hydraulic actuators can better handle linkages due to being able to exert a higher magnitude of force, however their footprint, and control complexity require larger and more rigid supporting structures, increasing the overall size of a set-up. SMA actuators can produce a relatively high actuation force and can be stacked closer together due to their small size. However, their actuation speed is low and they require highly efficient cooling systems, increasing the size footprint and the control complexity. Similarly, electromagnetic actuators also require cooling mechanisms for continuous usage and their stroke capacity is directly affected by additional weight from linkages. For specialized applications, piezoelectric actuators can be developed with a miniature footprint.

Interference must also be considered in a dense actuator configuration. It was already evident in our case study that electrical interference from data cables caused inaccuracies in potentiometer readings. Similarly, stacking electromagnets can cause magnetic interference. Cooling systems for SMA actuators can affect surrounding units, and must be targeted towards individual actuators, which increases control complexity.

Guideline 2: High-resolution shape-changing displays that require mechanical linkages would benefit from pneumatic and hydraulic techniques due to their ability to mitigate friction and weight issues caused by the linkages. However, electromechanical approaches are more practical as they are easier to configure.

\subsection{Physically Interactive and Passive Shape-Changing Applications.}

Physically interaction shape-changing applications requires the ability to physically move the actuator up and down as well as receive position feedback to detect the interactions so that a feature can be triggered (e.g. EMERGE - Taher et. al., 2015; inFORM - Follmer et. al., 2013). 
Pneumatic, electromagnetic, SMA, and electromechanical actuators with a belt drive can be used to support these interactions (e.g. through a mechanically connected part). Other electromechanical approaches, such as a lead screw (Hardy et. al., 2015) are more difficult to manually control. Although our electromagnetic lab prototype (as described in section 3.5.1) was based on an open-loop approach, using photo-reflectors (e.g. as used by Nakatani et. al., 2003) can provide closed-loop feedback.

Passive shape-changing applications simply show information and do not require user interaction (e.g. the Aegis Hyposurface by Goulthorpe et. al., 2001). All actuation techniques are suited to passive applications, and requirements are determined by specific application requirements. For instance, if fast transition between shapes is required, approaches such as pneumatic, electromechanical and electromagnetic are ideal. If the display needs to operate continuously, then electromagnetic and SMA techniques are less suitable as they have high heatoutput and require sophisticated cooling mechanisms. Finally, if granularity is important (the number of states an actuator can achieve, thus enabling a display to show more information), then any of the discussed techniques can, at a minimum, feasibly produce a medium granularity mechanism.

Guideline 3: Pneumatic, electromagnetic, SMA, and belt driven electromechanical actuators are ideal for physically interactive shape-changing applications that support attachments that users can push and pull.

Guideline 4: Passive displays require consideration of application-specific requirements to determine the suitability of actuators (e.g. electromechanical, pneumatic, and electromagnetic are ideal for applications that require fast transition between shapes). 


\subsection{Visual Output}

Visual output types in existing shape-changing displays typically involve projection (top and rear), LEDs, digital displays, and no visual output (in such cases the shape-displays serve as input devices or haptic feedback systems). Many shape-changing displays (e.g. FEELEX - Iwata et. al., 2001; Niiyama et. al., 2008; TAXEL - Kyung et. al., 2011) use projection on top of the actuators (usually with a flexible layer). The flexible layer provides a more organic interface for users, can also integrate sensor technology (Swallow and Thompson, 2001), and the use of projection enables a wide range graphics, as well as control over the resolution. However, interacting with a projected interface (e.g. touching the actuators) can occlude the graphics, especially in top-down projection techniques. EMERGE (Taher et. al., 2015) and LUMEN (Poupyrev et. al., 2004) utilize LED-based visual output. Displaying graphics on these interfaces is far more limited, but bars can be wholly illuminated (rather than just the top) without occlusion. Sublimate (Leithinger et. al., 2013) uses augmented reality, which allows graphics to be displayed in a $3 \mathrm{~d}$ space, however this requires additional equipment (e.g. augmented reality glasses, mobile device). Unlike mechanical actuators, fluid-based systems (pneumatic and hydraulic) can take advantage of transparent components to provide visual feedback. For example, fluid actuators can be placed on top of digital graphics displays (e.g. Tactus) to provide haptic feedback or show deformations (e.g. buttons or landscapes).

The visual output types described above are typically static and separate from the actuators, but approaches such as using digital displays or flexible displays can be integrated with the actuator itself. Tilt Displays (Alexander et. al., 2012), for instance, experiments with tilting a small-scale digital display, and MorePhone (Gomes, Nesbitt, and Vertegaal, 2013) uses a flexible E-ink display which can be deformed using SMA actuators. Combining actuated visual 
components can enhance the interactivity for shape-displays. For example, a shape-changing bar chart can have distinctive illuminated bars (through LEDs) with miniature displays that show the specific value of each bar.

Guideline 5: Visual output techniques such as projection on a flexible sheet placed on top of the actuators are simpler and allow more visual range. However, integrated visual output such as LEDs or OLEDs enable better tactile experience with individual actuating components.

\subsection{Actuator Power Requirements}

Input power requirements are an issue that is seldom discussed in past literature. While research prototypes are designed to explore application-specific factors, power requirements can determine the feasibility of using a large number of actuators in a high resolution display in a real-world scenario. For example, inFORM (Follmer et. al., 2013) consumes 300W for 100 actuators, EMERGE (Taher et. al., 2015) consumes 800W, and our hydraulic prototype consumes $264 \mathrm{~W}$ per single unit, making it highly impractical to construct a display with 100 hydraulic actuators. ShapeClip (Hardy et. al., 2015) explores portability through battery powered units, which can provide power for $\sim 30 \mathrm{~min}$. The portability of ShapeClip also allows each component to be easily removed (e.g. to replace a battery). In this case the battery life is short, however, exploring more efficient battery technologies is a useful way of reducing the power footprint and the control complexity.

Guideline 7: Electromechanical actuators typically consume the least amount of power in comparison to other techniques. However, shape-changing display research remains at an exploratory stage and investigating interaction and feasibility take precedence over, for instance, input power. Nevertheless, this creates an opportunity for further research in ways of reducing input power. 


\subsection{Significance of the Taxonomy}

This article has provided in-depth investigations into vertical actuation techniques and their role in shape-changing display applications through examining past work, constructing and detailing individual actuator prototypes, and describing a case study with a functional shape-display. These investigations validate the taxonomy and inform a set of guidelines (as described earlier in the Discussion section) that aims to aid HCI researchers in selecting actuators for shapechanging display applications. We therefore contribute original research and build on other works that have explored broader areas of shape-change. For instance, Rasmussen et. al. (2012) reviewed the design space of shape-changing displays and their transformative properties, Roudaut et al. (2013) explored a framework of shape transformations, Coelho and Zigelbaum (2011) surveyed shape-changing materials and explored soft material prototypes, and Hollerbach et al. (1992) examined the technical characteristics of various actuation techniques. Our investigations build on the above work by providing knowledge to HCI researchers about the technical (e.g. listing and discussing the implications of actuator speed, granularity, force, footprint, control, and feedback by constructing lab prototypes) and qualitative (e.g. reflecting on the development and usage of a fully constructed shape-display over a two-year period) properties of vertical actuators, in the context of shape-changing application development.

\subsection{Limitations and Generalization of the Taxonomy}

Our characterization was focused on vertical actuation, whereas shape-display research also includes other dynamic forms such as changes in volume and geometric deformations using soft materials. The actuators discussed, including the constructed lab prototypes reflects this focus; but we anticipate that these characteristics also apply to different configurations. Our case study 
is also limited to experiences with one actuation technique and application scenario, whereas different techniques and applications will create different challenges. Nevertheless, we believe that our characterization and reflections will allow researchers to better reason about actuator selection for shape-changing applications.

\section{Conclusion}

In this article we have presented a detailed investigation into the various characteristics of different actuation techniques used for vertical motion in shape-changing displays within the HCI community which, at present, is limited in exploration. These characteristics have been broken down and we present them as a taxonomy that is capable of describing the combination of features we see in the literature. Further, we validate the category breakdown through our own exploration via small-scale prototypes, through implications of their usage in shape-changing applications, and by describing a use-case scenario of a fully constructed and functional shapechanging display. Further, we have discussed that these individual characteristics cannot be considered in isolation, but instead must be used in parallel with each other when selecting or creating an actuator design. These are supported by design guidelines to aid researchers in selecting actuation techniques and to promote the discussion of design requirements and tradeoffs for developing shape-changing applications. This work provides an original contribution that builds on previous characterization work in shape-changing displays that has focused on the general transformative, material, and technical characteristics of shape-change. As a result, this article aids the rapid development of novel shape-changing interactions by providing in-depth and reflective descriptions and discussions of vertically moving actuators. 


\section{Acknowledgements}

This work forms part of GHOST, a project funded by the European Commission's 7th Framework Programme, FET-open scheme (grant \#309191).

\section{References}

Alexander, J., Lucero, A., and Subramanian, S. (2012). Tilt displays: designing display surfaces with multi-axis tilting and actuation. Mobile HCI, 161-170.

Chauvelin, C., Sagi, T., Coni, P., André, J. M., Jauze, C., and Lespinet-Najib, V. (2014). Haptics on a touch screen: characterization of perceptual thresholds. International Journal of HumanComputer Interaction, 30(11), 872-881.

Coelho, M., Ishii, H., and Maes, P. (2008). Surflex: a programmable surface for the design of tangible interfaces. CHI $E A$.

Coelho, M., and Maes, P. (2008). Sprout I/O: a texturally rich interface. TEI, 221-222.

Coelho, M., and Zigelbaum, J. (2011). Shape-changing interfaces. Personal and Ubiquitous Computing, 15, 161-173.

Follmer, S., Leithinger, D., Olwal, A., Hogge, A., and Ishii, H. (2013). inFORM: dynamic physical affordances and constraints through shape and object actuation. UIST, 417-426.

Frey, M. (2004). SnOil-A Physical Display Based on Ferrofluid.

Frisken-Gibson, S. F., Bach-y-Rita, P., Tompkins, W. J., and Webster, J. G. (1987). A 64Solenoid, Four-Level Fingertip Search Display for the Blind. IEEE BME, 34(12), 963-965. 
Gomes, A., Nesbitt, A., and Vertegaal, R. (2013). MorePhone: a study of actuated shape deformations for flexible thin-film smartphone notifications. $C H I$, 583-592.

Goulthorpe, M., Burry, M., and Dunlop, G. (2001). Aegis hyposurface: The bordering of university and practice. of ACADIA, 344-349.

Hardy, J., Weichel, C., Taher, F., Vidler, J., and Alexander, J. (2015) ShapeClip: Towards Rapid Prototyping with Shape-Changing Displays for Designers. CHI, 19-28.

Harrison, C., and Hudson, S. E. (2009). Providing dynamically changeable physical buttons on a visual display. CHI, 299-308.

Hernandez, H., Preza, E., and Velazquez, R. (2009). Characterization of a Piezoelectric Ultrasonic Linear Motor for Braille Displays. CERMA pp.402-407.

Hirota, K., and Hirose, M. (1995). Simulation and presentation of curved surface in virtual reality environment through surface display. VRAIS, 211-216.

Hollerbach, J.M., Hunter, I.W., and Ballantyne, J. (1992). A comparative analysis of actuator technologies for robotics. The robotics review, vol. 2, 299-342, August 1992.

Iwata, H., Yano, H., Nakaizumi, F., and Kawamura, R. (2001). Project FEELEX: adding haptic surface to graphics. SIGGRAPH, 469-476.

Jansen, Y., Karrer, T., and Borchers, J. (2010). MudPad: tactile feedback and haptic texture overlay for touch surfaces. ITS, 11-14. 
Kyung, K. U., Kwon, D. S., and Yang, G. H. (2006). A novel interactive mouse system for holistic haptic display in a human-computer interface. International Journal of HumanComputer Interaction, 20(3), 247-270.

Kyung, K., Lim, J. M., Lim, Y., Park, S., Park, S. K., Hwang, I., Choi, S., Seo, J., Kim, S., Yang, T., and Kwon, D. (2011). TAXEL: Initial progress toward self-morphing visio-haptic interface. WHC, 37-42.

Leithinger, D., Follmer, S., Olwal, A., Luescher, S., Hogge, A., Lee, J., and Ishii, H. (2013). Sublimate: State-changing virtual and physical rendering to augment interaction with shape displays. $C H I, 1441-1450$.

Leithinger, D., Lakatos, D., Devincenzi, A., Blackshaw, M., and Ishii, H. (2011). Direct and Gestural Interaction with Relief : A 2 .5D Shape Display. UIST, pp.541-548.

Nakatani, M., Kajimoto, H., Vlack, L., Sekiguchi, D., Kawakami, N., and Tachi, S. (2005). Control method for a 3D form display with coil-type shape memory alloy. In Proc. ICRA, 13321337.

Nakatani, M., Kajimoto, H., and Sekiguchi, D. (2003). 3D Form Display with Shape Memory Alloy. ICAT, 179-184.

Niiyama, R., and Kawaguchi, Y. (2008). Gemotion Screen: A Generative, Emotional, Interactive 3D Display. In Proc. ASIAGRAPH, 115-120.

Oersted, H. C. (1820). Electricity and magnetic needles. Philosophy, 16(4), 273-276. 
Poupyrev, I., Nashida, T., Maruyama, S., Rekimoto, J., and Yamaji, Y. (2004). Lumen: interactive visual and shape display for calm computing. In ACM SIGGRAPH Emerging Technologies, 17.

Poupyrev, I., Nashida, T., and Okabe, M. (2007). Actuation and tangible user interfaces: the Vaucanson duck, robots, and shape displays. TEI, 205-212.

Poupyrev, I., Rekimoto, J., and Maruyama, S. (2002). TouchEngine: a tactile display for handheld devices. CHI EA, 644-645.

Rasmussen, M., Pedersen, E., Petersen, M., and Hornbæk, K. (2012). Shape-changing interfaces: a review of the design space and open research questions. $C H I, 735-744$.

Rekimoto, J., and Schwesig, C. (2006). PreSenseII: bi-directional touch and pressure sensing interactions with tactile feedback. CHI EA, 1253-1258.

Roudaut, A., Karnik, A., Lochtefield, M., and Subramanian, S. (2013). Morphees: toward high shape resolution in self-actuated flexible mobile devices. $C H I, 593-602$.

Swallow, S. S., and Thompson, A. P. (2001). Sensory fabric for ubiquitous interfaces. International Journal of Human-Computer Interaction, 13(2), 147-159.

Taher, F., Hardy, J., Karnik, A., Weichel, C., Jansen, Y., Hornbaek, K., and Alexander, J. (2015). Exploring Interactions with Physically Dynamic Bar Charts. CHI, 3237-3246.

Tsimeris, J., Dedman, C., Broughton, M., and Gedeon, T. (2013). ForceForm: A Dynamically Deformable Interactive Surface. In Proc. ITS, 175-178. 
Wagner, C. R., Lederman, S. J., and Howe, R. D. (2002). A tactile shape display using RC servomotors. HAPTICS, 354-355.

Zhu, H., and Book, W.J. (2004). Practical structure design and control for digital clay. In Proc. ASME, 1051-1058. 
Table 1: Classification of the actuators used in shape-changing applications in past literature and in our constructed prototypes using the taxonomy. The classification key described in the taxonomy section is in brackets. We estimate granularity based on control system setup where it is not explicitly stated.

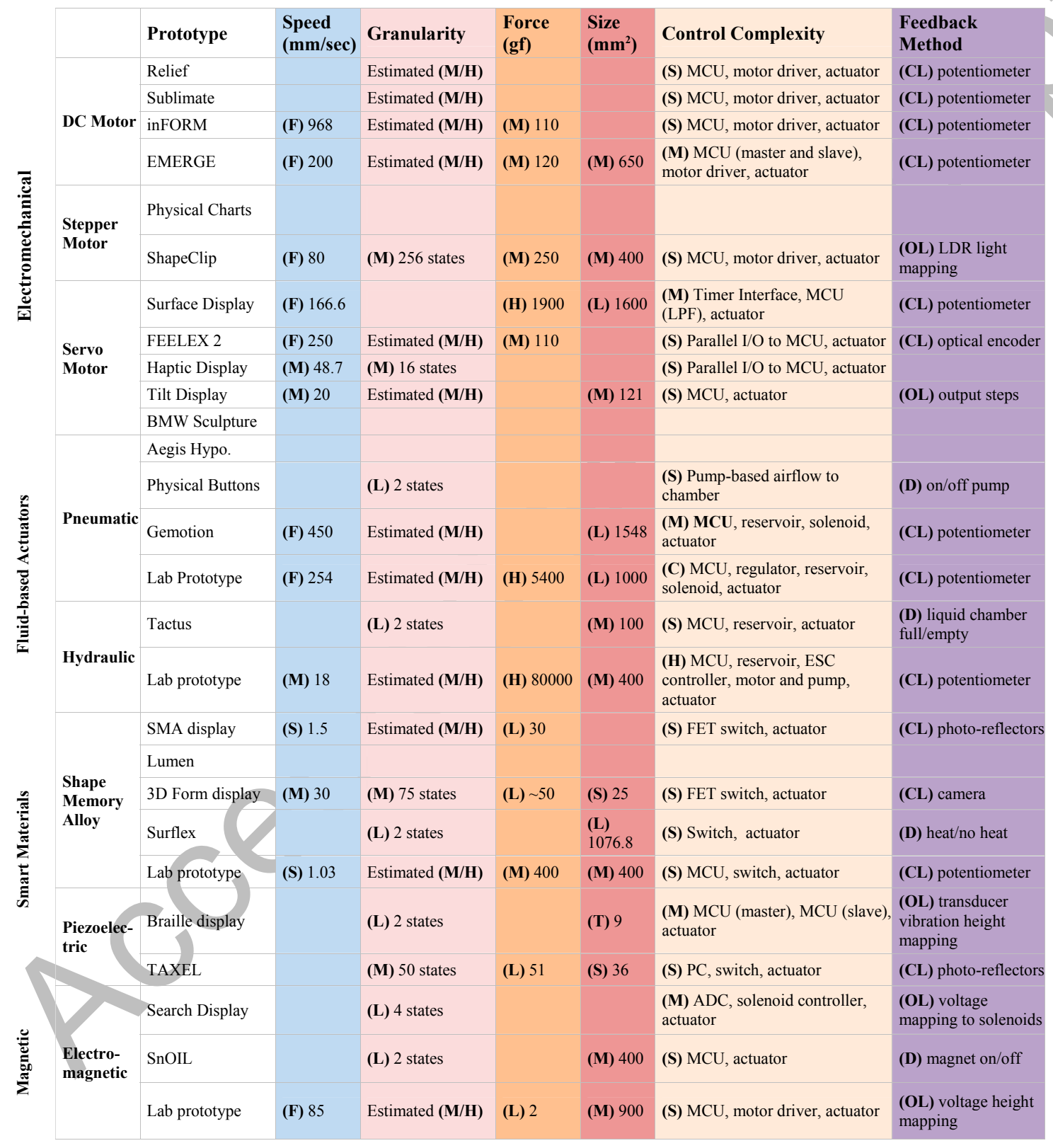


Figure 1: (Left) The configuration of the DC motor actuator. (Right) The DC motor actuator prototype.

Figure 2: (Left) The stepper motor actuator configuration. (Right) The stepper motor prototype.
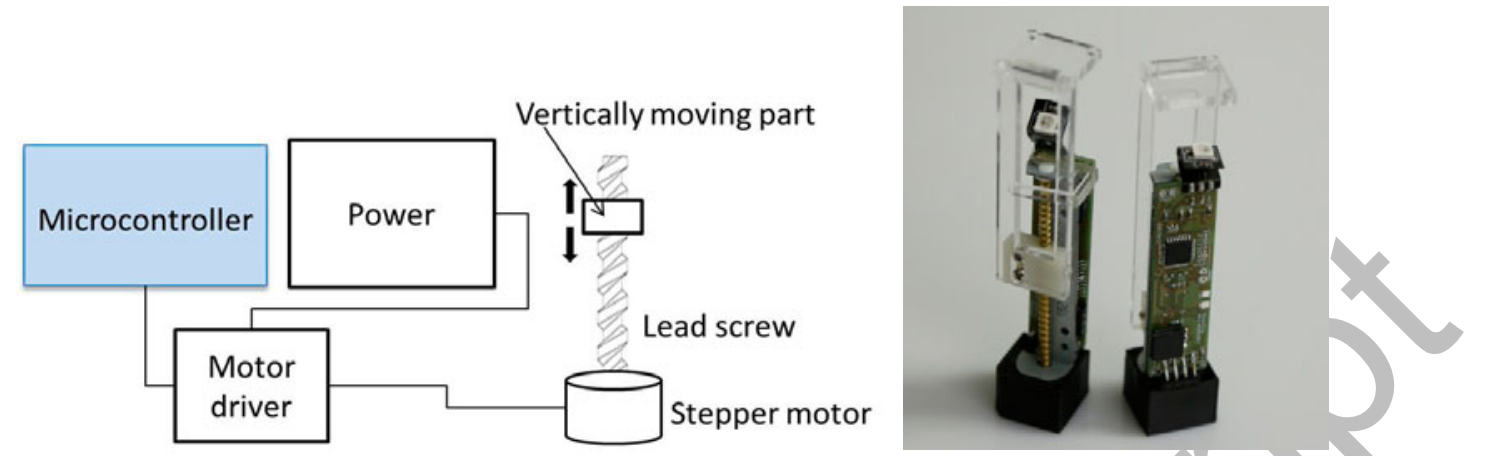
Figure 3: (Left) The configuration of the pneumatic actuator. (Right) The pneumatic actuator prototype.
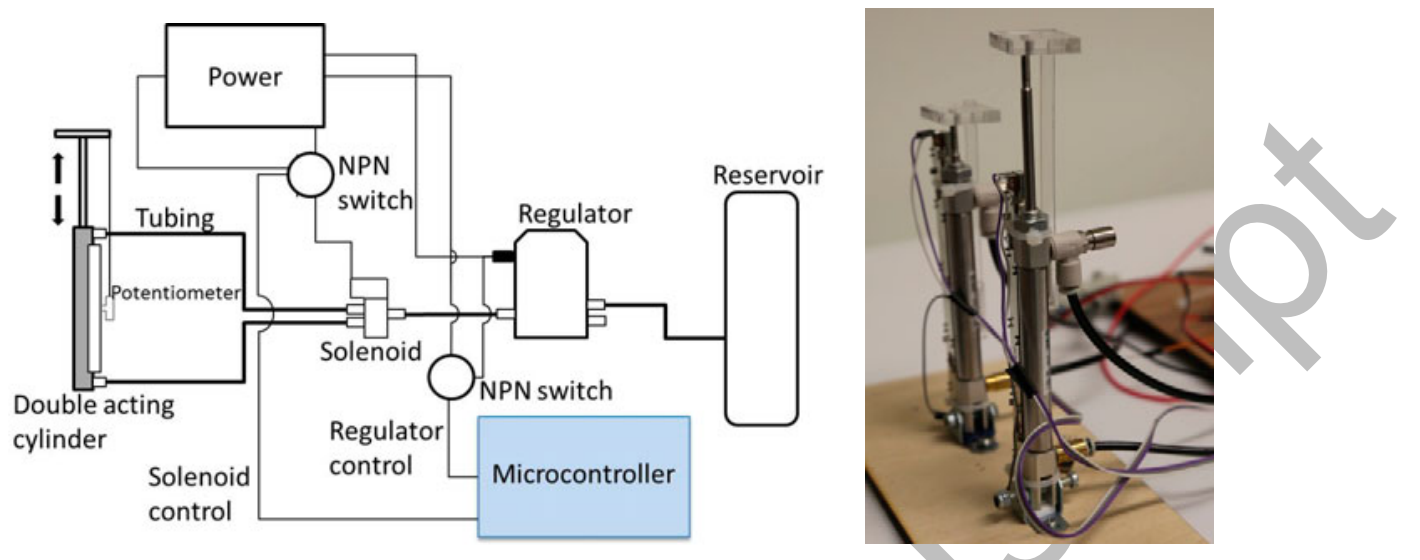
Figure 4: (Left) The configuration of the hydraulic actuator. (Right) The hydraulic actuator prototype.
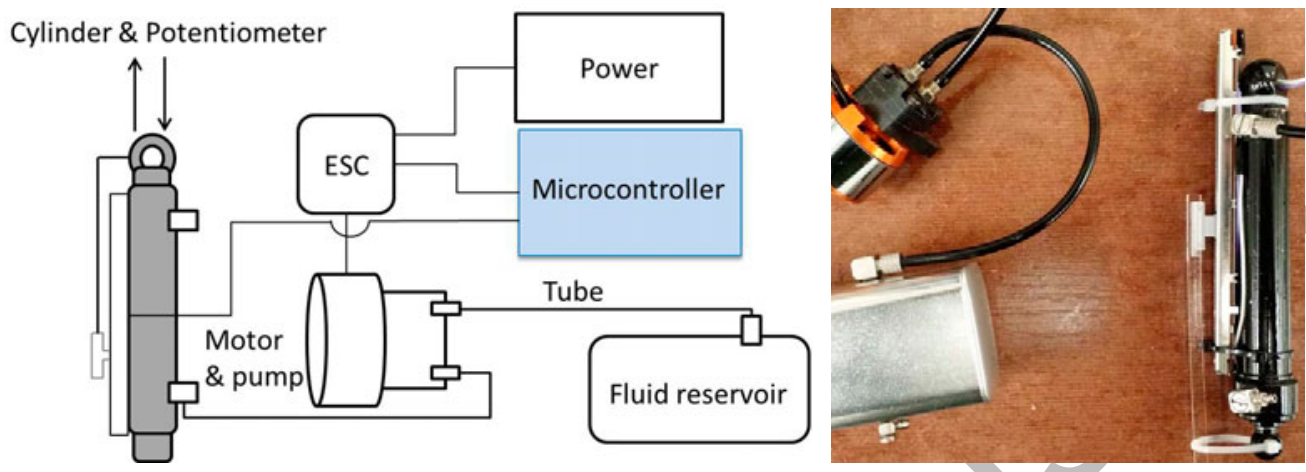
Figure 5: (Left) The configuration of the Shape-memory alloy (SMA) actuator. (Right) The SMA actuator prototype.
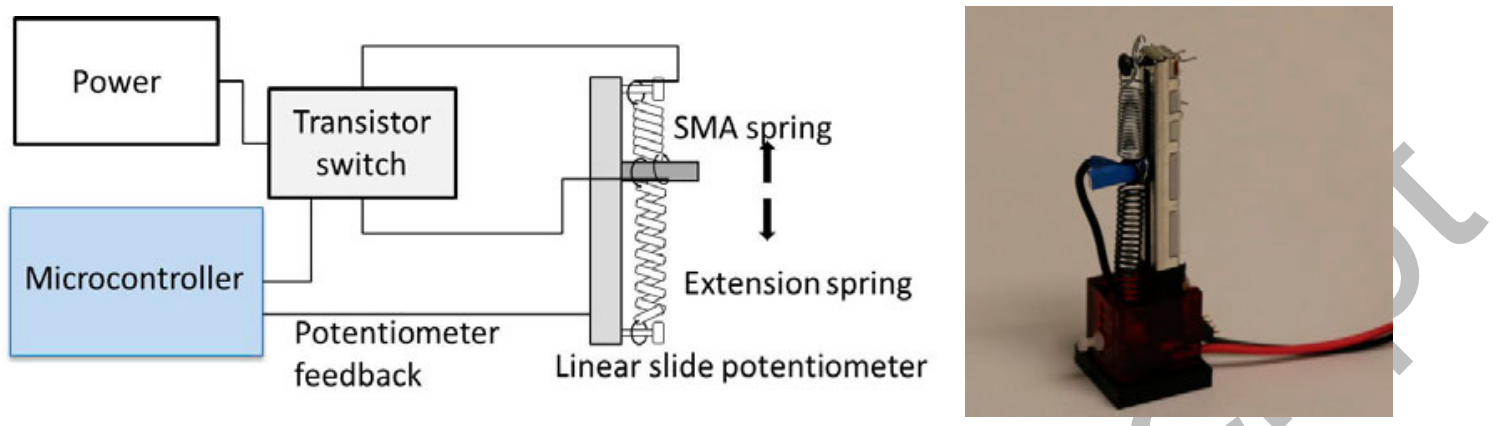
Figure 6: (Left) The configuration of the electromagnetic actuator. (Right) The electromagnetic actuator prototype.

Figure 7: (Left) The configuration of the $10 \times 10$ shape-changing bar chart. (Right) The shapechanging bar chart prototype.

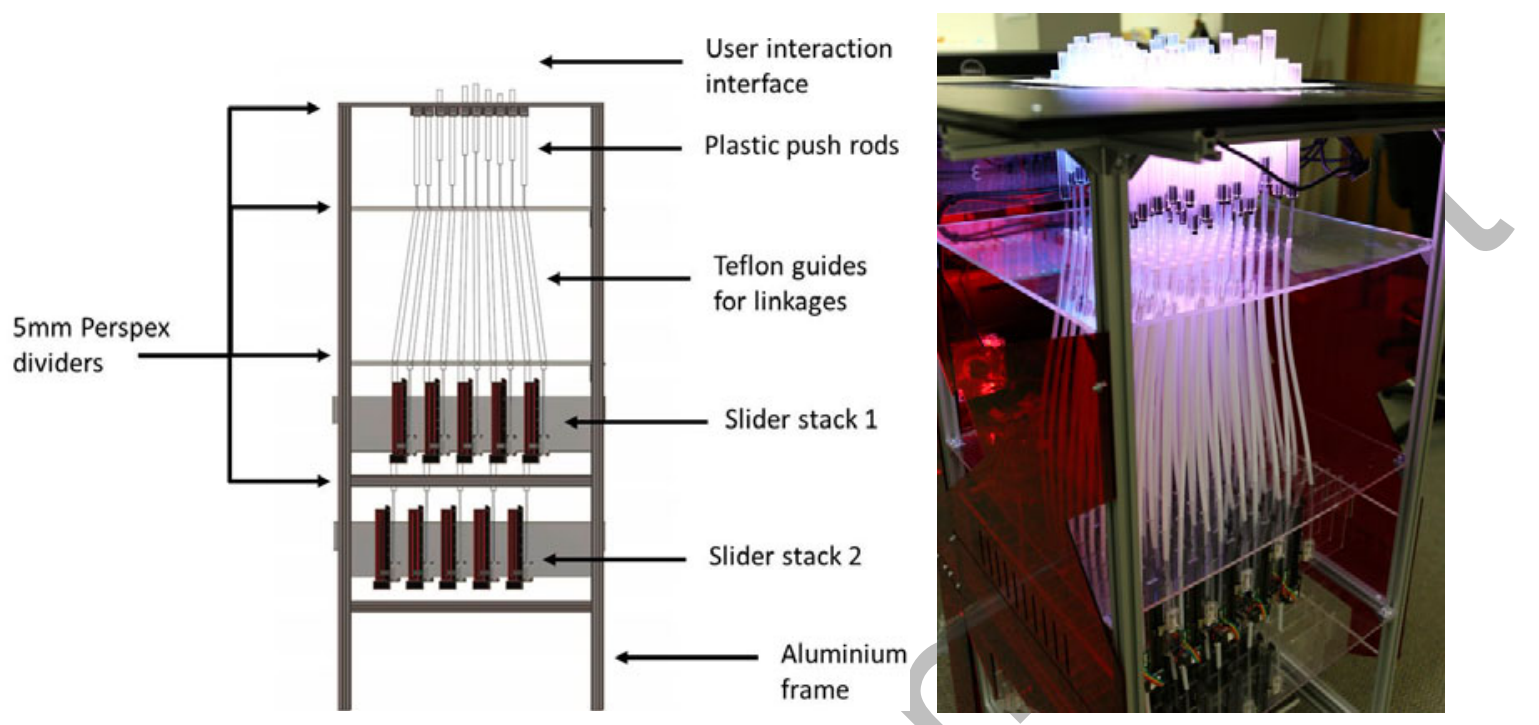




\section{Author Biographies}

\section{Faisal Taher}

Dr. Faisal Taher is a Research Associate in the School of Computing and Communications at Lancaster University, UK. His work focuses on building and evaluating shape-changing display prototypes, that is, displays with physically reconfigurable geometry. This forms part of his wider interest in the field of Human-Computer Interaction.

\section{John Vidler}

John Vidler is a PhD student in the School of Computing and Communications at Lancaster University, UK. His work mainly focuses on communication between low level systems and the hardware/software boundary at the operating systems level. He uses his varied experience with electronics, computing and mechanical systems to do this.

\section{Jason Alexander}

Dr. Jason Alexander is a Senior Lecturer in Human-Computer Interaction in the School of Computing and Communications at Lancaster University, UK. His work addresses computing's physical-digital divide through the development of novel interaction techniques, devices, and displays. He does this by combining expertise in hardware prototyping and empirical user evaluation. 